\title{
Pipeline Bonded Joints Assemble and Operation Health Monitoring with Embedded FBG Sensors ${ }^{\dagger}$
}

\author{
Thiago Destri Cabral 1,2,*, Antonio Carlos Zimmermann ${ }^{2}$, Daniel Pedro Willemann ${ }^{3}$ and \\ Armando Albertazzi Gonçalves, Jr. ${ }^{2}$ \\ 1 Laboratory of Photonic Materials \& Devices (LIQC), School of Mechanical Engineering, University of \\ Campinas, São Paulo 13083-860, Brazil \\ 2 Laboratory of Metrology and Automation (LABMETRO), Federal University of Santa Catarina, Santa \\ Catarina 88040-970, Brazil; ac.zimmermann@labmetro.ufsc.br (A.C.Z.); a.albertazzi@ufsc.br (A.A.G.J.) \\ 3 Mechanics, Machines and Motors Laboratory (Lab3M), Santa Catarina State University, \\ Santa Catarina 8879-000, Brazil; daniel.willemann@udesc.br \\ * Correspondence: tdcabral@ifi.unicamp.br; Tel.: +55-19-3521-5423 \\ + Presented at the 7th International Electronic Conference on Sensors and Applications, 15-30 November \\ 2020; Available online: https://ecsa-7.sciforum.net/.
}

Published: 15 November 2020

\begin{abstract}
Offshore oil and gas platforms present a harsh environment for their installed infrastructure, with pipelines that are both subjected to a corrosive atmosphere and transport aggressive chemicals being the most critical. These conditions have prompted the industry to substitute metallic pipelines for composite counterparts, often made of fiber reinforced plastics assembled with bonded joints. Various technologies have emerged in the recent years to assess the health of these composite pipelines. In particular, robust speckle metrology techniques such as shearography, although not capable of long-term monitoring, have produced very satisfactory results. However, these inspection techniques require specialized equipment and trained personnel to be flown to offshore platforms, which can incur in non-trivial inspection costs. In this paper we propose and demonstrate a robust and cost-effective approach to monitor pipelines bonded joints during assembly and operation using Fiber Bragg Grating (FBG) sensors embedded into the joints' adhesive layer. This approach allows for informed decisions on when to perform targeted in-depth inspections (e.g., with shearography) based on both real-time and long-term feedback of the FBG sensors data, resulting on lower monitoring costs, a severe increase on monitoring uptime (up to full uptime), and increased operational security.
\end{abstract}

Keywords: optical fiber sensors; fiber reinforced plastics; composite pipelines; structural health monitoring; fiber Bragg gratings; oil and gas pipelines; tubular bonded joints

\section{Introduction}

In applications where crucial system components are frequently or constantly in contact with corrosive substances, such as in the oil and gas industry, usage of engineering plastics and composite materials has grown exponentially, due to low weight-to-strength ratio and high corrosion resistance inherent to these materials. Metallic pipelines, for example, are being replaced by adhesive bonded fiber reinforced plastic (FRP) pipelines generating a demand for FRPs inspection techniques. Several NDT techniques have been adapted or developed to meet this demand, including thermography, ultrasonography, radiography, holography and computed tomography [1]. In previous publications we have explored the use of a robust speckle pattern shearing interferometry technique, shearography, with success for assessing the health of bonded joints in FRP pipelines [2-4]. The downside of these techniques is that access to the inspection site and components to be inspect is often difficult, as is for 
pipelines that are buried or installed in confined spaces on oil platforms. Furthermore, specialized equipment and trained personnel need to be flown to offshore platforms, which can incur in nontrivial inspection costs and reduce monitoring uptime.

Fiber optic sensors, and in particular Fiber Bragg Gratings (FBGs) are increasingly popular for structural health monitoring applications due to advantageous characteristics that are inherent to fiber sensors, such as reduced size (typical fiber diameter of $125 \mu \mathrm{m}$ ), immunity to electromagnetic interference and high multiplexing capability [5]. While embedding sensors in plastics and composite materials has been a highly active field of study in recent years, the use of these techniques applied to tubular bonded joints is a very recent topic and remain a significant technical challenge. [6-8].

In this work we explore the use of FBGs embedded into the adhesive layer of tubular adhesive bonded joints for long-term structural health monitoring and defect detection throughout the entire lifecycle of the pipeline. This was achieved by embedding several sensing fibers in bonded joints between industrial chlorinated polyvinylchloride (CPVC) pipes to measure axial strain inside the adhesive and looking for anomalies in the strain fields that indicate the presence of defects.

\section{Materials and Methods}

\subsection{Instrumentation Setup}

The standard FBG sensor consists of a short section of optical fiber with a periodically modulated refractive index. When broadband light is launched into the fiber, the FBG structure couples the forward-propagating light to a backwards core mode, creating a narrowband reflection response. The peak wavelength of the reflected signal, $\lambda_{B}$ (Bragg wavelength) is sensitive to mechanical strain and temperature experienced by the fiber, as shown in Equation (1), where $\varepsilon$ is the mechanical strain along the axis of the fiber, $T$ temperature, and $k_{\varepsilon}$ and $k_{T}$ are the strain and temperature sensitivity respectively (typically $k_{\varepsilon}=0.769$ and $k_{T}=7.64 \times 10^{-6} \mathrm{~K}^{-1}$ ). Thus, the FBG can be used as a strain sensor by monitoring the shift of $\lambda_{B}$ in response to mechanical loads [6,7].

$$
\Delta \lambda_{B} / \lambda_{B}=k_{\varepsilon} \Delta \varepsilon+k_{T} \Delta T
$$

In this work we have employed sensing fibers with 3 FBG strain sensors along their length. Precautions in the form of a polyimide protective coating and a $0.9 \mathrm{~mm}$ diameter Teflon loose tube were used to prevent fiber breakage during handling, embedding, and testing. A schematic drawing of the used sensing fibers can be seen in Figure 1.

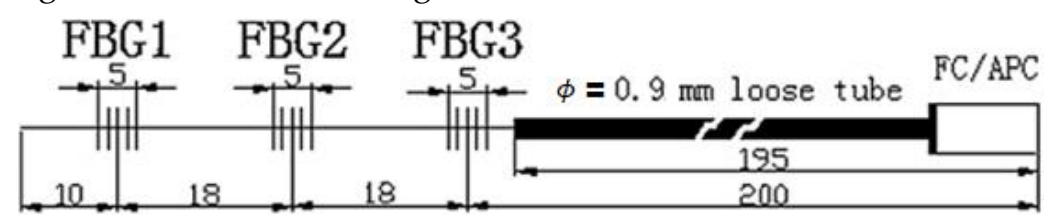

Figure 1. Sensing fiber with 3 FBGs, loose tube and FC/APC connector. Dimensions in millimeters.

Distinct nominal $\lambda_{B}$ were used for each FBG, along with sufficient spectral spacing ( $\geq 5 \mathrm{~nm}$ ) to easily distinguish between sensors and avoid signal overlapping from uneven loading. Furthermore, each embedded fiber has a unique combination of nominal wavelengths, so each sensing location along the perimeter of a bonded joint is uniquely and readily identifiable.

\subsection{Assembly of Test Specimens}

The test specimens were assembled with 4" $(100 \mathrm{~mm})$ nominal diameter schedule 80 CPVC pipe and fittings. One end of the specimen is sealed with and endcap, while the opposing end is fitted with a heavy-duty CPVC flange and sealed with a 1040 steel blind flange with a central 1/4" NPT thread. Both the endcap and CPVC flange were solvent welded to straight pipe sections, which were then adhesive-bonded to a sleeve using bi-component methyl-methacrylate adhesive.

To accommodate the sensing fibers and allow for bonding the straight pipe sections to the sleeve, the length of pipe to be inserted into the sleeve $(\sim 58 \mathrm{~mm})$ was machined to reduce its diameter by 
$3 \mathrm{~mm}$. Figure 2 is a picture of a fully assembled test specimen with embedded sensors. Note that in this configuration FBG1 (Figure 1) will be positioned the deepest into the sleeve, while FBG3 is positioned closer to the outer edge of the sleeve (where the fiber exits the adhesive layer), with FBG2 in between.

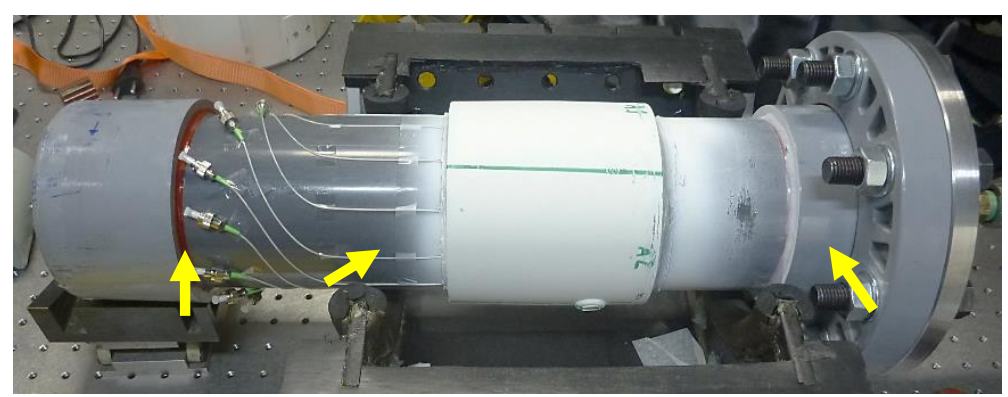

Figure 2. Fully assembled test specimen ready for hydrostatic testing. Yellow arrows indicate the points were the test specimen is supported by the fixtures below it.

Breakage of the sensing fibers while assembling the bonded joints was avoided by first prepositioning the fiber with adhesive tape (applied to the loose tube), securing the tip of the fiber to the wall of pipe with a droplet of cyanoacrylate adhesive, and then applying a uniform layer of the methyl-methacrylate bi-component adhesive directly along the length of the fiber before applying it to the machined pipe section and inner wall of the sleeve. Finally, the instrumented pipe section and sleeve are carefully brought together, avoiding rotation along their long axis as to not misalign the fibers. Three test specimens, that will be referred as S1, S2 and S3, were assembled. Specimens S2 and S3 are instrumented with 12 sensing fibers in the adhesive layer $(\sim 43.6 \mathrm{~mm}$ of circumferential separation between fibers) aligned with the pipe's long axis, providing 36 strain sensing points, whereas $\mathrm{S} 1$ has 11 fibers ( $\sim 7.6 \mathrm{~mm}$ circumferential spacing) due to a defective fiber.

\section{Results and Discussion}

Specimen S1 was assembled with a significant lack of adhesive to create numerous large voids, while S2 was prepared to be as structurally sound as possible. S3 contains planned defects created by embedding pieces of Teflon sheet with aluminum tape (to increase detectability by tomography). The instrumented joints were then submitted to a computed tomography scan for control purposes, the results are depicted in Figure 3 along with a picture of the Teflon pieces embedded into S3.

(a)

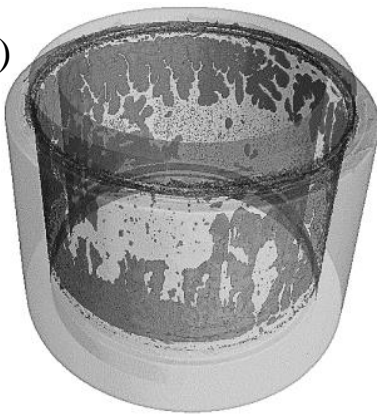

(b)

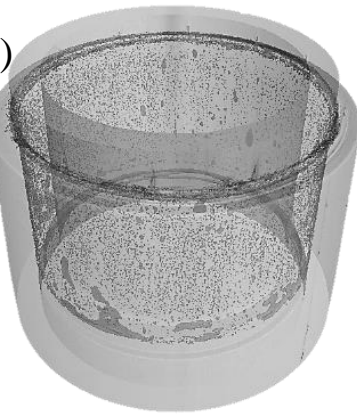

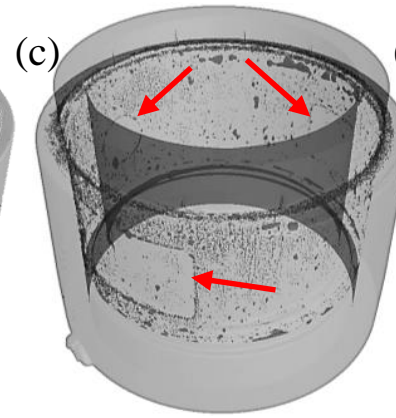

Figure 3. (a-c): Computed tomography images of the instrumented bonded joints from S1, S2 and S3, respectively. Red arrows indicate the position of the artificial inclusions (d) in S3.

From Figure 3a extensive lack-of-adhesive defects (darker areas) can be seen in S1, some porosity without major voids in S2 (Figure 3b), and some porosity along the planned defects (indicated by the red arrows) in S3 (Figure 3c). The embedded defects shown in Figure 3d have the approximate dimensions of $20 \mathrm{~mm}$ diameter for the circle, $20 \mathrm{~mm}$ sides for the square and $20 \mathrm{~mm}$ by $70 \mathrm{~mm}$ for the rectangular Teflon sheet. For a more complete evaluation of the instrumentation setup defect detection capacity the circle and large rectangle were positioned on top of FBG sensors, while the 
square was placed in the spacing between fibers. Three experiments were conducted with the embedded FBG sensors to assess their suitability for monitoring the health of the bonded joints during all stages of the pipeline lifecycle: (a) adhesive curing strain test (joint assembly), (b) flexural strain detection test (pipeline assembly), and (c) hydrostatic test (operation).

Adhesive curing strain test was realized by interrogating (sm125 Optical Sensing Interrogator, 1 pm resolution-Micron Optics) all FBGs before and after assembling the bonded joints and computing the strain difference $(\Delta \varepsilon)$ between datasets. Figure 4 contains the $\Delta \varepsilon$ maps (surface and color maps with bilinear interpolation, note that in the graphics the axis start at zero and as such FBG0 in the graph = FBG1 in Figure 1 and so on) of the adhesive cure strain for S1 and S2. In a structurally sound joint the strain map is expected to be entirely negative (compressive strain) as the adhesive shrinks when cured [9]. This is visible in the $\Delta \varepsilon$ maps for S2, along with great circumferential symmetry (Optical Fiber axis) and a higher strain magnitude towards the outer edge of the sleeve. On the other hand, S1 exhibits significant strain fluctuations due to the severe lack of adhesive, no circumferential symmetry and FBGs under tensile strain. Therefore, it is evident that the embedded sensors can differentiate between a sound joint and one with moderate to severe lack of adhesive.
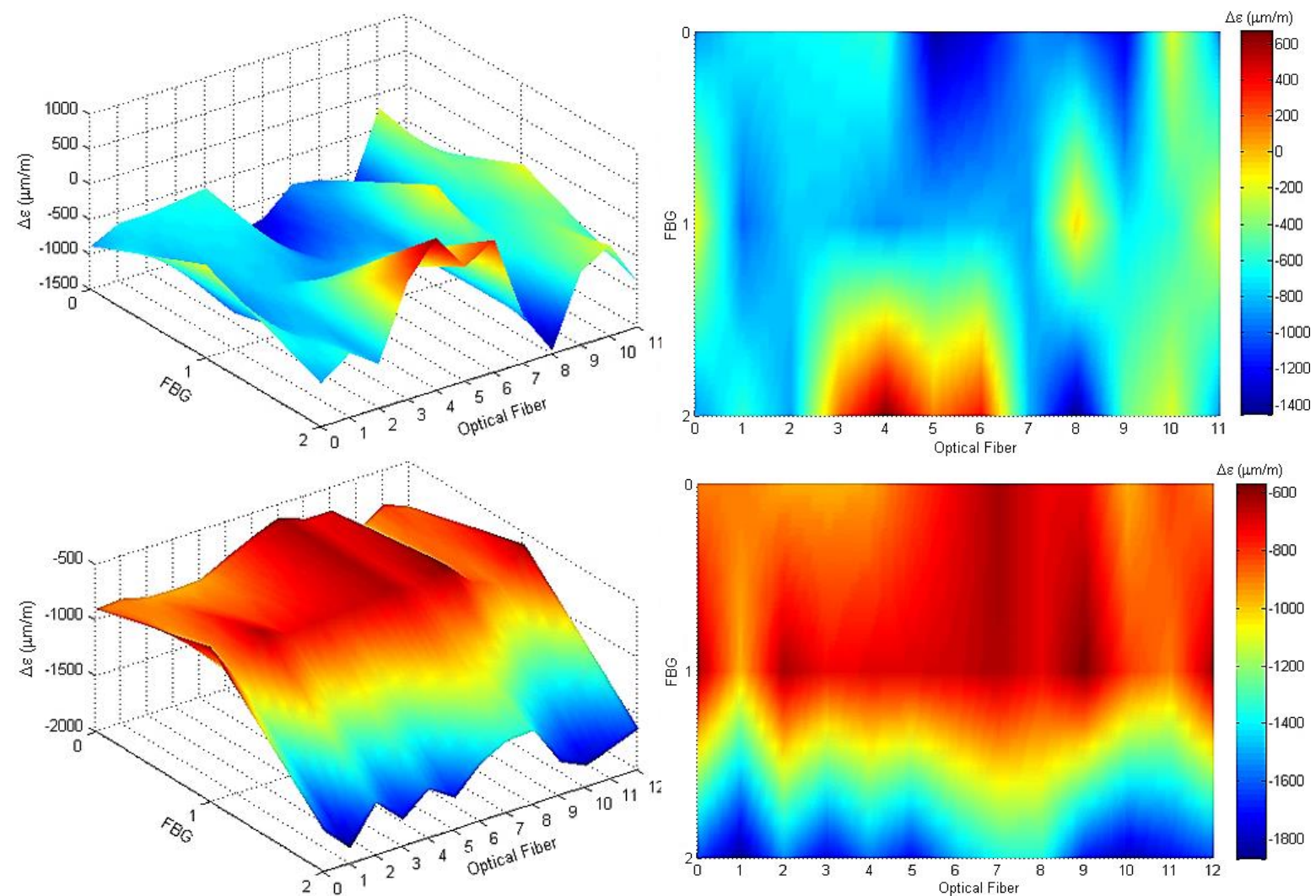

Figure 4. Adhesive cure strain for S1 (top), S2 (bottom). Left: surface plots; Right: color plots.

When assembling pipelines, forcefully compensating for misalignments between pipe sections to be joined can introduce axial and flexural loads, leading to premature failures due to increased strain levels. As indicated by the yellow arrows in Figure 2, when ready for hydrostatic testing the test specimens are supported in 3 points: the endcap and two points near the sleeve. The two-point support at both sides of the sleeve results in a slight flexural load with a maximum at the midpoint of the sleeve. In this configuration, by comparing strain maps obtained before and after rotating the test specimen along the long axis, the load redistribution among sensing fiber simulates the effect of introducing a slight flexural load. The strain difference $(\Delta \varepsilon)$ map for a "virtual" load, obtained by rotating S2 by $90^{\circ}$ is shown in Figure 5. As can be seen, the strain distribution is consistent with uniaxial flexural loading, the FBGs are also shown to be very sensitive, being able to detect very slight flexural loads, undoubtedly adequate for monitoring the pipeline assembly process. 

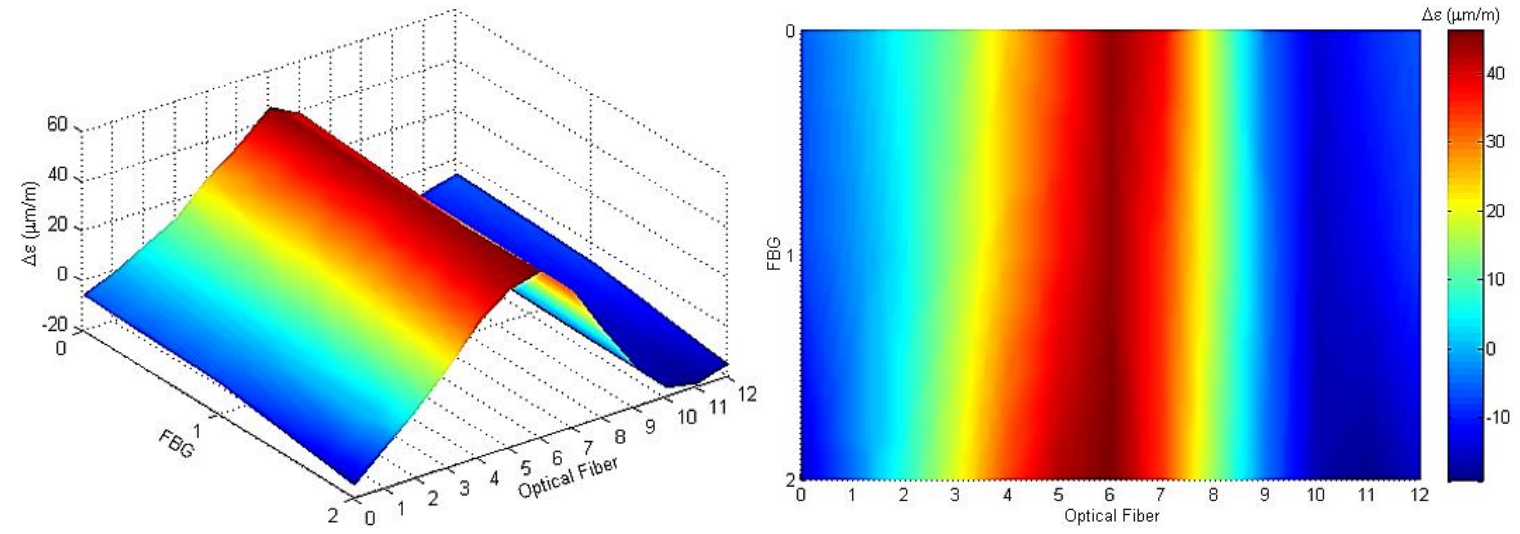

Figure 5. Surface and color plots of the $\Delta \varepsilon$ map obtained by rotating S2 by $90^{\circ}$ along the long axis.

Finally, hydrostatic testing was conducted to evaluate the defect detection and monitoring effectiveness of the embedded sensors when the pipeline is pressurized during operation. The test specimens were pressurized up to 7 bar $(0.7 \mathrm{MPa})$ manometric pressure using a hydraulic hand pump. Figure 6 contains strain maps obtained by computing the strain difference $(\Delta \varepsilon)$ detected by all FBG sensors at the 7 bar internal pressure in relation to the unpressurized (zero manometric pressure) reference strain values. As expected for this type of test, the $\Delta \varepsilon$ map for S2 (Figure 6a) shows purely tensile strain, as the sealed pipe is forced to elongate due to the internal pressure load. It is noteworthy that strain levels are reasonably uniform (mean strain $=79.5 \mu \varepsilon$, standard deviation $=13.4 \mu \varepsilon$ ), with the fluctuations most likely caused by porosity (observed in Figure 3b) near the FBGs.
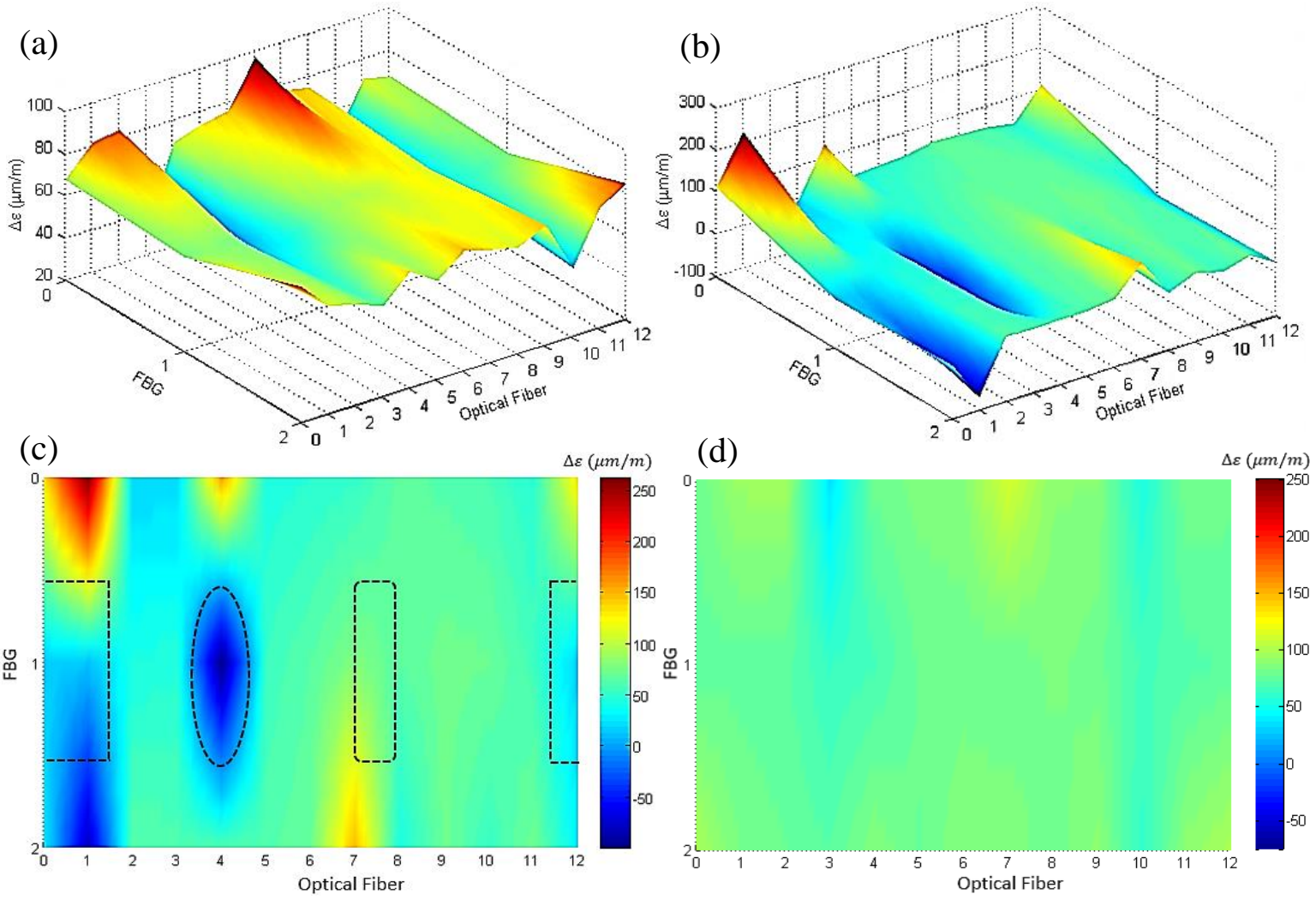

Figure 6. (a) and (b): Hydrostatic test $\Delta \varepsilon$ map surface plots for S2 and S3. (c): Color plot of the $\Delta \varepsilon$ map in (b), dashed black lines mark the position of intentionally embedded defects. (d): Color plot of the $\Delta \varepsilon$ map in (a) with the color bar rescaled to match with (c). Internal pressure $=7$ bar.

The strain map for S3 in its turn (Figure 6b,c) shows very distinct anomalies: a dip into negative strain levels is observed coinciding with the position of the circular embedded defect (elongated in Figure $6 \mathrm{c}$ due to the map aspect ratio), and a noticeable strain redistribution is observed in the region 
containing the larger rectangular defect. The smaller square defect, which was purposefully positioned away from the FBG sensors, unfortunately is not observable it did not cause a large enough disturbance in the strain field to be detected by surrounding FBGs. If the color bar for the strain map of S2 is rescaled to match that of S3 (Figure 6d), the difference between the strain map of a sound bonded joint and one with embedded defects becomes very evident. When compared to the strain concentration and redistribution (including areas under compression) caused by the presence of defects (Figure 6c), the strain fluctuations observed before in Figure 6a are very small, rendering the rescaled strain colormap extremely smooth. This comparison clearly shows that the embedded sensors can detect and monitor the presence of defects during the pipeline operation.

\section{Conclusions}

A sensing setup to detect and monitor defects in bonded joints between pipelines by measuring axial strain in the adhesive layer with embedded FBG sensors was demonstrated. This setup was shown to be adequate to monitor the assembly of the joints, assembly of the pipelines, and the pipeline operation, effectively covering all stages of the pipeline lifecycle. Although further studies are needed before practical field measurements can be realized, for example the developing strategies for compensating temperature effects on the FBG sensors and determining the optimal sensor density (fibers per joint) to ensure defects of critical dimensions do not remain undetected, the achieved results of this work are very promising and can find ample use for monitoring plastic and composite pipelines that make use of adhesive bonded joints.

Author Contributions: T.D.C., D.P.W. and A.A.G. conceived the original idea, T.D.C assembled the test specimens and performed the experiments; T.D.C. wrote the paper, A.C.Z., D.P.W. and A.A.G. gave funding and instrumentation support. Authorship must be limited to those who have contributed substantially to the work reported.

Funding: This research was funded by Petróleo Brasileiro S.A. (PETROBRAS) and Conselho Nacional de Desenvolvimento Científico e Tecnológico (CNPq).

Conflicts of Interest: The authors declare no conflict of interest.

\section{References}

1. Wang, B.; Zhong, S.; Lee, T.-L.; Fancey, K.S.; Mi, J. Non-destructive testing and evaluation of composite materials/structures: A state-of-the-art review. Adv. Mech. Eng. 2020, 12, 1-28, doi:10.1177/1687814020913761.

2. Willemann, D.P.; Fantin, A.; Gonçalves, A.A., Jr. Defect assessment of bonded joints of composite tubes using shearography. In Speckle 2010: Optical Metrology; International Society for Optics and Photonics: 2010; doi:10.1117/12.870680.

3. Willemann, D.P.; Silva, F.A.; Benedet, M.E.; Fantin, A.V. Applications of a Compact NDT Shearography System Specially Designed for the Petroleum and Gas Industry. In Proceedings of the 27th ASNT Research Symposium, Orlando, FL, USA, 26 March 2018; doi:10.32548/RS.2018.003.

4. Macedo, F.J.; Benedet, M.E.; Fantin, A.V.; Willemann, D.P.; Da Silva, F.A.A.; Albertazzi, A. Inspection of defects of composite materials in inner cylindrical surfaces using endoscopic shearography. Opt. Lasers Eng. 2018, 104, 100-108, doi:10.1016/j.optlaseng.2017.06.005.

5. Majumder, M.; Gangopadhyay, T.K.; Chakraborty, A.K.; Dasgupta, K.; Bhattacharya, D. Fibre Bragg gratings in structural health monitoring-Present status and applications. Sensors Actuators A Phys. 2008, 147, 150-164, doi:10.1016/j.sna.2008.04.008.

6. Canal, L.P.; Sarfaraz, R.; Violakis, G.; Botsis, J.; Michaud, V.; Limberger, H.G. Monitoring strain gradients in adhesive composite joints by embedded fiber Bragg grating sensors. Compos. Struct. 2014, 112, 241-247, doi:10.1016/j.compstruct.2014.02.014.

7. Chen, Y.; Hsieh, C.C.; Lin, C. Strain measurement for composite tubes using embedded, fiber Bragg grating sensor. Sensors Actuators A Phys. 2011, 167, 63-69, doi:10.1016/j.sna.2011.02.035.

8. Kang, H.-K.; Kang, D.-H.; Bang, H.-J.; Hong, C.-S.; Kim, C.-G. Cure monitoring of composite laminates using fiber optic sensors. Smart Mater. Struct. 2002, 11, 279-287, doi:10.1088/0964-1726/11/2/311. 
9. Kosaka, T.; Osaka, K.; Sawada, Y. Evaluation of Residual Strain of Fibres in Matrix Resin during Cure Process by Optical Fibre Sensors. In Proceedings of the ICCM-17-17th International Conference on Composite Materials, Edinburgh, UK, 27-31 July 2009.

Publisher's Note: MDPI stays neutral with regard to jurisdictional claims in published maps and institutional affiliations.

(C) 2020 by the authors. Licensee MDPI, Basel, Switzerland. This article is an open access article distributed under the terms and conditions of the Creative Commons Attribution (CC BY) license (http://creativecommons.org/licenses/by/4.0/). 